\title{
X-ray binaries as the origin of nebular He II emission in low-metallicity star-forming galaxies
}

\author{
D. Schaerer ${ }^{1,2}$, T. Fragos ${ }^{1}$, and Y. I. Izotov ${ }^{3}$ \\ 1 Observatoire de Genève, Université de Genève, 51 Ch. des Maillettes, 1290 Versoix, Switzerland \\ e-mail: daniel.schaerer@unige.ch \\ 2 CNRS, IRAP, 14 Avenue E. Belin, 31400 Toulouse, France \\ ${ }^{3}$ Bogolyubov Institute for Theoretical Physics, National Academy of Sciences of Ukraine, 14-b Metrolohichna str., \\ Kyiv 03143, Ukraine
}

Received 2 January 2019 / Accepted 17 January 2019

\begin{abstract}
The origin of nebular He II emission, which is frequently observed in low-metallicity $(\mathrm{O} / \mathrm{H})$ star-forming galaxies, remains largely an unsolved question. Using the observed anticorrelation of the integrated X-ray luminosity per unit of star formation rate $\left(L_{X} / \mathrm{SFR}\right)$ of an X-ray binary population with metallicity and other empirical data from the well-studied galaxy I Zw 18 , we show that the observed He II $\lambda 4686$ intensity and its trend with metallicity is naturally reproduced if the bulk of $\mathrm{He}^{+}$ionizing photons are emitted by the X-ray sources. We also show that a combination of X-ray binary population models with normal single and/or binary stellar models reproduces the observed $I(4686) / I(\mathrm{H} \beta)$ intensities and its dependency on metallicity and age. We conclude that both empirical data and theoretical models suggest that high-mass X-ray binaries are the main source of nebular He II emission in low-metallicity star-forming galaxies.
\end{abstract}

Key words. galaxies: ISM - galaxies: high-redshift - X-rays: binaries

\section{Introduction}

Since its discovery, the origin of nebular He II $\lambda 4686$ emission observed in (giant) H II regions, H II galaxies, dwarf galaxies, Wolf-Rayet galaxies, and similar sources that are known or generally thought to be powered by stellar radiation (e.g., Pakull \& Angebault 1986; Garnett et al. 1991; Guseva et al. 2000; Shirazi \& Brinchmann 2012) has remained mysterious. The nebular He II emission lines (the He II $\lambda 4686$ or the He II $\lambda 1640 \mathrm{UV}$ line) in the integrated spectrum indicate the existence of sources of hard ionizing radiation, that is, photons with energies above $54 \mathrm{eV}$ (or $\lambda<228 \AA$, corresponding to the ionization potential of $\left.\mathrm{He}^{+}\right)$.

Only stars with very high effective temperatures $T_{\text {eff }} \gtrsim$ 80-100 kK emit non-negligible amounts of $\mathrm{He}^{+}$ionizing photons (e.g., Tumlinson \& Shull 2000; Schaerer 2002). Because such temperatures are only reached in very peculiar evolutionary phases (e.g., in the Wolf-Rayet (WR) or planetary nebula phase), "normal" stellar populations contain few such stars. Their ionizing spectra are therefore in general not hard enough to explain the observed nebular He II $\lambda 4686$ emission, with typical intensities $I(4686) / I(\mathrm{H} \beta)$ of a few percent (e.g., Izotov \& Thuan 2004). Exceptions are populations of very low metallicity $\left(\lesssim 10^{-4}\right.$ solar) or zero metallicity (PopIII; cf. Schaerer 2003), which are not observed, however. Alternatively, the observed nebular He II emission can be explained by the presence of WR stars in some galaxies with metallicities $12+\log (\mathrm{O} / \mathrm{H}) \gtrsim 8.4$ (e.g., Schaerer 1996; Shirazi \& Brinchmann 2012). However, only a small fraction of nebular He II emitters show WR stars, and the intensity of $\mathrm{He}$ II $\lambda 4686$ increases with decreasing metallicity (cf. also below), opposite to that of the WR populations (Guseva et al. 2000). For all these reasons, photoionization models using ionizing spectra predicted from "standard" stellar populations fail to reproduce the observed nebular He II emission, especially at low metallicities, $12+\log (\mathrm{O} / \mathrm{H}) \lesssim 8.2$, where it is most prominent (cf. Shirazi \& Brinchmann 2012; Stasińska et al. 2015).

Several studies have explored the impact of various physical processes, such as stellar rotation and binarity, on the evolution of massive stars, predicting for instance the existence of hotter single main-sequence stars in cases of very fast rotation leading to quasi-homogeneous evolution (see, e.g., Szécsi et al. 2015; Maeder 1987), and "rejuvenated", hot stars formed in interacting binary systems (e.g., van Bever \& Vanbeveren 1998; Eldridge et al. 2017; Götberg et al. 2018), both of which imply hotter stellar populations on average and hence a harder ionizing spectrum. However, none of these works has so far been able to quantitatively explain the observed intensity of the He II emission in low-metallicity galaxies. For example, the latest BPASS binary population and spectral synthesis models incorporating the effect of binary mass transfer on stellar evolution falls short of predicting the observed He II $\lambda 4686 / \mathrm{H} \beta$ intensities by typically one order of magnitude, even considering different stellar initial mass functions (see Stanway \& Eldridge 2019, and below).

Since the first discoveries of He II emitters, other sources and processes that could emit more energetic photons than stellar sources have been suggested. These include X-ray binaries (XRBs), photoionization by X-rays, and strong shocks. X-rays can explain specific cases, but appear insufficient in others (e.g., Pakull \& Angebault 1986; Garnett et al. 1991; 
Thuan \& Izotov 2005; Kehrig et al. 2015). For some galaxies it is argued that shocks can explain He II emission, if they provide $\sim 10 \%$ of the hydrogen ionizing photons (e.g., Thuan \& Izotov 2005; Izotov et al. 2012). However, no straightforward predictive model that would allow linking shock models to other galaxy properties exists, and it is not clear how shocks would reproduce the observed trends of He II intensity with metallicity (cf. below). In short, the origin of nebular He II emission in star-forming galaxies is so far considered an unsolved question, with numerous important implications, also for our understanding of distant galaxies (e.g., Cassata et al. 2013; Stark 2016; Steidel et al. 2016; Vanzella et al. 2016; Berg et al. 2018).

Here we present a new approach to this question, considering observational and empirical findings from X-rays and optical spectroscopy, insight from detailed modeling of the lowmetallicity galaxy I Zw 18, and recent models of XRB populations. We examine in particular the implications of the increased importance of X-ray emission in low-metallicity galaxies (e.g., Kaaret et al. 2011; Basu-Zych et al. 2013; Douna et al. 2015; Brorby et al. 2016) on the He II problem. We show that massive $\mathrm{X}$-binaries and/or ultra-luminous X-rays sources (ULX), which are a natural extension of the latter to higher X-ray luminosities, are able to reproduce the basic observed trends and are therefore the most likely sources of nebular He II $\lambda 4686$ emission in low-metallicity star-forming galaxies.

\section{Comparing observed scalings of nebular He ॥ $\lambda 4686$ emission and X-rays}

\subsection{Star-forming galaxy samples}

Low-metallicity $(12+\log (\mathrm{O} / \mathrm{H}) \lesssim 8.4)$ galaxies, where nebular $\mathrm{He}$ II is frequently detected, exhibit one main observational behavior that we show in Fig. 1: a clear trend of increasing line intensity $I(4686) / I(\mathrm{H} \beta)$ with decreasing metallicity. To illustrate the He II observations, we here use a compilation of more than 1400 star-forming galaxies or regions thereof from Izotov et al. (2016), which are selected for the quality of the spectra, allowing direct metallicity determinations via the classical auroral line method $^{1}$. The $I(4686) / I(\mathrm{H} \beta)$ detections follow a sub-linear scaling with metallicity (with a slope of $\sim-0.45$ ), as indicated by the yellow line. Our aim is not to properly determine a mean relation for this quantity taking completeness and other factors into account. Our goal is instead to show how "typical" sources and observed trends with metallicity can be reproduced.

Interestingly, the analysis of the X-ray emission of starforming galaxies shows that the X-ray luminosity, $L_{X}$, normalized per star formation rate (SFR), also increases with decreasing metallicity with a similar dependence as the He II intensity. Douna et al. (2015) obtained a near-linear scaling of $L_{X} /$ SFR with $\mathrm{O} / \mathrm{H}$, whereas Brorby et al. (2016) found a somewhat shallower dependence. Both studies showed an increase of $L_{X} / \mathrm{SFR}$ by more than one order of magnitude from solar metallicity to the lowest metallicity X-ray detected galaxies, such as I Zw 18 at $\sim 1 / 30$ solar $(12+\log (\mathrm{O} / \mathrm{H})=7.18$, Izotov \& Thuan 1998).

The empirical relations between $L_{X} / \mathrm{SFR}$ and $\mathrm{O} / \mathrm{H}$ can easily be translated into a relation between the $I(4686) / I(\mathrm{H} \beta)$ intensity ratio and $\mathrm{O} / \mathrm{H}$. We only need to assume or determine one parameter, the average $\mathrm{He}^{+}$ionizing photon flux per $L_{X}$ of the sources

1 The sources include 1135 sources from the Sloan Digital Sky Survey (SDSS), and 328 are from observations obtained by Izotov and collaborators with 4-8 m class telescopes. Standard emission line ratios were used to select star-forming galaxies, not AGN.

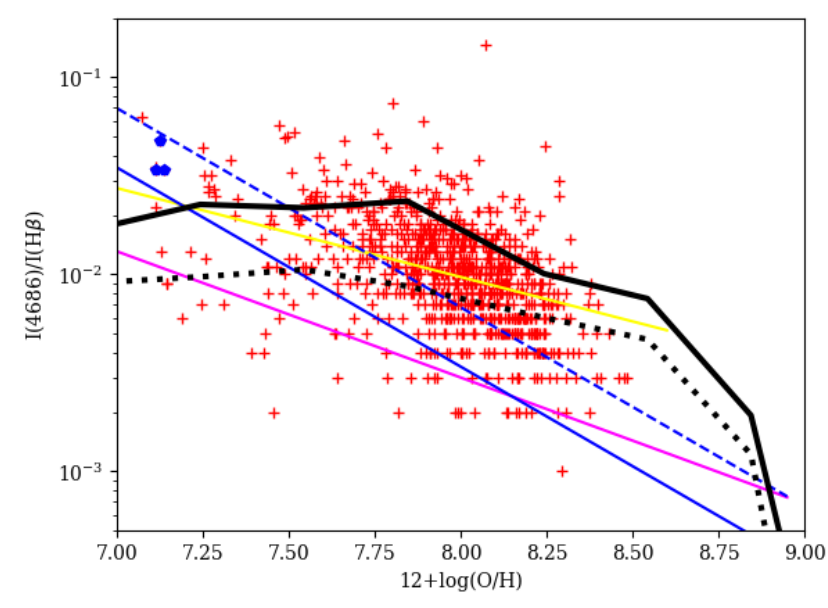

Fig. 1. Observed and predicted $I(4686) / I(\mathrm{H} \beta)$ relative nebular line intensities as a function of metallicity. Observations of low-metallicity star-forming galaxies are shown as red plusses, and different measurements of the NW region of I Zw 18 with blue diamonds. The yellow line shows the linear regression to the data points. Assuming a default value of $q=2 \times 10^{10}$ photon $\mathrm{erg}^{-1}$, the empirical $L_{X} / \mathrm{SFR}-\mathrm{O} / \mathrm{H}$ relations of Douna et al. (2015) and Brorby et al. (2016) translate into He II intensities shown by the blue and magenta solid lines, respectively. The black lines show the predicted $\mathrm{He}$ in intensity adopting $L_{X}$ /SFR predicted from the XRB synthesis models described in Sect. 3 for a constant SFR over $10 \mathrm{Myr}$ (dotted) and $0.1 \mathrm{Gyr}$ (solid), and the same value of $q$. The blue dashed line differs from the solid line by assuming a value of $q$ that is a factor of two higher.

responsible for the X-ray luminosity

$q=Q\left(\mathrm{He}^{+}\right) / L_{X}$.

Given the proportionality between the Lyman continuum photon flux and the SFR, that is, taking $Q(\mathrm{H}) / \mathrm{SFR}=9.26 \times$ $10^{52}$ photon s$^{-1} /\left(M_{\odot} \mathrm{yr}^{-1}\right)$ (Kennicutt 1998), this allows us to derive the ratio of $\mathrm{He}^{+}$over $\mathrm{H}$ ionizing photons, $Q\left(\mathrm{He}^{+}\right) / Q(\mathrm{H})$, which directly yields the observed He II $\lambda 4686$ line intensity

$I(4686) / I(\mathrm{H} \beta)=A \times Q\left(\mathrm{He}^{+}\right) / Q(\mathrm{H})$,

where $A \approx 1.74$ for typical nebular conditions (see, e.g., Stasińska et al. 2015). Here we have assumed that the SFR is constant over $\gtrsim 10 \mathrm{Myr}$, which is consistent with the assumption of even longer SF timescales ( $2100 \mathrm{Myr}$ ) made for the empirical SFR determinations that were used by the above studies in determining $L_{X} /$ SFR.

The He II intensity as a function of metallicity predicted from the empirical $L_{X} / \mathrm{SFR}$ versus $\mathrm{O} / \mathrm{H}$ relations of Douna et al. (2015) and Brorby et al. (2016) assuming $q=$ $2 \times 10^{10}$ photon $\mathrm{erg}^{-1}$ (cf. below) are shown in Fig. 1. We suggest that the close similarity of the dependence of $L_{X} / \mathrm{SFR}$ and $I(4686) / I(\mathrm{H} \beta)$ on metallicity also indicates a causal connection between them, that is to say, that X-ray sources are the dominant source of $\mathrm{He}^{+}$ionizing photons. We now examine I Zw18, one of the best-studied low-metallicity galaxies, in particular, to estimate $q$ empirically.

\subsection{Zw 18 as a testbed}

The low-metallicity dwarf galaxy I Zw 18 has been studied in detail. It is known to show nebular He II emission concentrated in the NW region with an integrated intensity of $I(4686) / I(\mathrm{H} \beta) \sim 0.02-0.04$ (e.g., Izotov \& Thuan 1998; 
Kehrig et al. 2015; Lebouteiller et al. 2017). The NW region, a giant $\mathrm{H}$ II region, also harbors a point-like $\mathrm{X}$-ray source that dominates the observed X-ray emission of this galaxy and is suggested to be a single XRB (Thuan et al. 2004; Kaaret \& Feng 2013). A point-source luminosity $L_{X}=3 \times 10^{39} \mathrm{erg} \mathrm{s}^{-1}$ in the $0.5-10 \mathrm{keV}$ range has been determined by Thuan et al. (2004) from Chandra observations, whereas Kaaret \& Feng (2013) find a higher $L_{X}=1.4 \times 10^{40} \mathrm{erg} \mathrm{s}^{-1}$ from XMM data, possibly indicating variability according to these authors.

Using the vast set of multiwavelength observations, Lebouteiller et al. (2017) have recently constructed a tailored model of the neutral and ionized interstellar matter (ISM) of this galaxy. Based on their detailed photoionization modeling including stellar radiation, emission from an accretion disk model that successfully fit the X-ray source, and cosmic rays, they were able to reproduce all the observational constraints. Their model shows in particular that both the emission lines of low- and high-ionization stages, including also He II, which are strongly affected by high-energy radiation, are well explained by the observed X-ray source in this galaxy. A successful physical model including ionization from a stellar black hole was also constructed by Heap et al. (2019). From the observed properties of I $\mathrm{Zw} \mathrm{18,} \mathrm{we} \mathrm{can} \mathrm{therefore} \mathrm{empirically} \mathrm{derive} \mathrm{the} \mathrm{amount} \mathrm{of}$ $\mathrm{He}^{+}$ionizing photons emitted from the X-ray source, assuming that the latter dominates the ionizing photon flux above $54 \mathrm{eV}$.

Using the observations of value of Kehrig et al. (2015), who find $Q\left(\mathrm{He}^{+}\right)=1.33 \times 10^{50}$ photon s$^{-1}$, and the two values of the X-ray luminosities reported above, we thus obtain $q=Q\left(\mathrm{He}^{+}\right) / L_{X}=(1.0-3.4) \times 10^{10}$ photon $\mathrm{erg}^{-1}$. Adopting an intermediate value of $q=2 \times 10^{10}$ photon $\operatorname{erg}^{-1}$, we find that the observed $I(4686) / I(\mathrm{H} \beta)$ intensity of $\mathrm{I} \mathrm{Zw} 18$ is well reproduced from the $L_{X} / \mathrm{SFR}$ relation of Douna et al. (2015), as shown in Fig. 1. This is indeed expected because I Zw 18 lies perfectly on their mean relation. However, the bulk of the observations are better reproduced with a higher value of $q=$ $4 \times 10^{10}$ photon $^{-1}$, as also shown in this figure.

\section{Nebular He II $\lambda 4686$ emission from X-ray binary population models}

We now explore how XRB population synthesis models compare with the nebular He II $\lambda 4686$ observations. To do this we use models developed by Fragos et al. (2013a,b) to study the cosmological evolution of XRB populations that were recently recalibrated to updated measurements of the cosmic star-formation history and metallicity evolution (Madau \& Fragos 2017). The same type of models and choice of model parameters has been shown to produce XRB populations that are consistent with observation of both the local and the distant universe (e.g., Tzanavaris et al. 2013; Tremmel et al. 2013; Lehmer et al. 2016). An important feature of the models is the strong dependence of the XRB population on metallicity, both in terms of the formation efficiency of XRBs and the integrated X-ray luminosity of the whole population.

The predictions of the integrated X-ray luminosity per unit of SFR from the synthetic models as a function of stellar population age and metallicity for simple stellar populations (bursts) are shown in Fig. 2. The X-ray luminosity corresponds to the $0.3-10 \mathrm{keV}$ range and absorption from a hydrogen column density of $N_{\mathrm{H}}=3 \times 10^{21} \mathrm{~cm}^{-2}$ (Madau \& Fragos 2017).

Assuming, for example, $S F R=$ const over a period of $0.1 \mathrm{Gyr}$, we find an increase of $I(4686) / I(\mathrm{H} \beta)$ by more than order of magnitude between solar metallicity and $12+\log (\mathrm{O} / \mathrm{H}) \sim 7.6$, and a flattening thereafter, as shown in Fig. 1. For shorter periods of

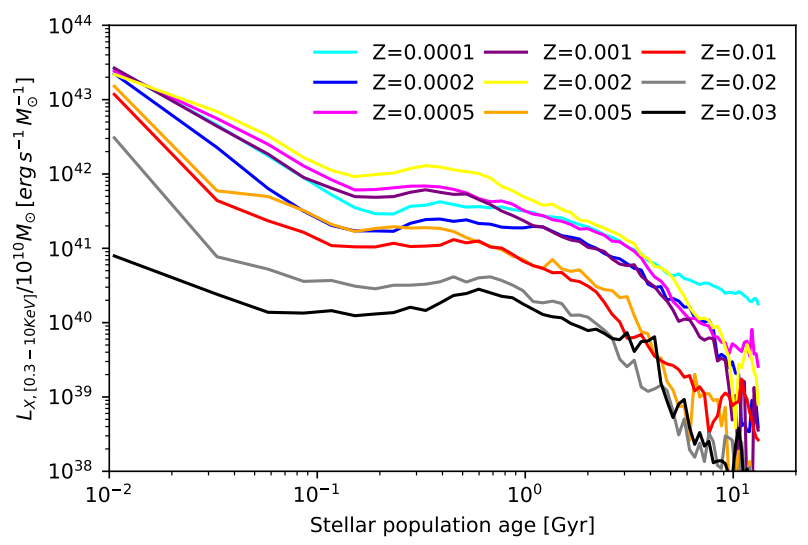

Fig. 2. Predicted X-ray luminosity as a function of age for simple stellar populations with different metallicities.

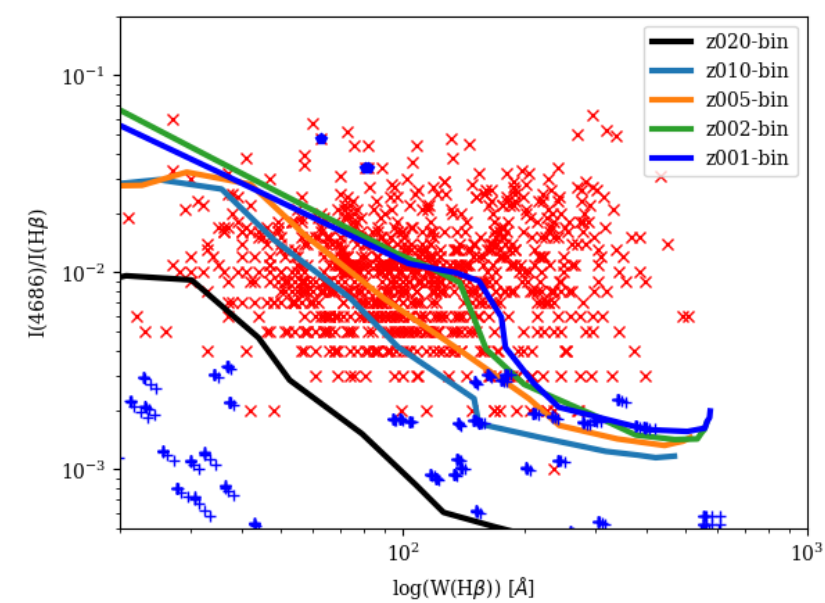

Fig. 3. Observed and predicted $I(4686) / I(\mathrm{H} \beta)$ relative nebular line intensities as a function of the $\mathrm{H} \beta$ equivalent width. Observations are the same as in Fig. 1. Blue crosses show the predictions from the BPASS models of Xiao et al. (2018), which fail to reproduce the observed He II $\lambda 4686$ intensities. Predictions for simple stellar populations of different metallicities (shown by the solid lines), derived from the combination of BPASS + XRB models, show a fair agreement with the observations.

constant SFR the result is very similar; for longer periods the He II intensity may be somewhat higher. In any case, the models follow both the observed line intensity and its metallicity dependence quite well. Here the predicted values of $L_{X} / \mathrm{SFR}$ have been translated into the observed $I(4686) / I(\mathrm{H} \beta)$ intensity using the same simple assumptions we described above, that is, with one adjusted parameter $(q)$.

Because the He II intensity is also expected to depend on the age of stellar population, we examine in Fig. 3 its dependence on the $\mathrm{H} \beta$ equivalent width, $W(\mathrm{H} \beta)$, a well-known proxy for age in young simple stellar populations (SSPs). To do so we combine the predictions from the BPASSv2.1 synthesis models, describing the evolution of an ensemble of single and binary stars as a function of age, with that of the XRB population resulting from a stellar population with the same total mass. BPASS yields the predicted temporal evolution of $Q(H)$ and $W(\mathrm{H} \beta)$, whereas the XRB model predicts $L_{X}(t)$, from which derive $Q\left(\mathrm{He}^{+}\right)$from Eq. (1), hence $I(4686) / I(\mathrm{H} \beta)$.

As shown in Fig. 3, the SSP models including X-ray binaries show an increase of $I(4686) / I(\mathrm{H} \beta)$ by more than one order of magnitude compared to the BPASS models, which include 
interacting binaries, but not the XRB phase. The models cover the range of observed He II intensities and are in fair agreement with most of the data. Possible disagreements remain for the galaxies with the highest $W(\mathrm{H} \beta) \gtrsim 200 \AA$, which correspond to ages younger than $\lesssim 5 \mathrm{Myr}$ according to the BPASS models used here. This would suggest that luminous X-ray sources appear fairly soon to explain He II emission from these galaxies as well. This is consistent with detailed binary evolutionary models, which show that the first ULX sources may turn on as early as $\sim 4 \mathrm{Myr}$ after a burst of star formation (e.g., see Fig. 4 in Rappaport et al. 2005). Alternatively, X-ray sources from an underlying older population (e.g., with a constant SFR, cf. Fig. 1), or shocks (cf. Izotov et al. 2012, 2018) could also contribute to boost the He II emission in these sources.

We conclude that when we include the contribution of XRBs in our stellar population models and making a simple assumption on the $\mathrm{He}^{+}$ionizing photon flux emitted by the $\mathrm{X}$-ray sources, we are able to generally reproduce the observed nebular He II emission in star-forming galaxies at low metallicity, although some difficulties may exist in explaining the youngest sources.

\section{Discussion}

\subsection{Non-detections and scatter}

In our compilation, approximately two-thirds of the spectra show nebular He II $\lambda 4686$ emission, with no indication for a dependence of the detection fraction on metallicity. While the empirical scaling relations and the simple models described above explain the presence and strength of He II, we also need to consider the cases of He II $\lambda 4686$ of non-detections. There are many possible explanations for this, for example, stochasticity effects related to the XRB population, variability of the $\mathrm{X}$-ray sources, and variations in the assumed mean value of $q$ (Eq. (1)). Stochasticity related to the low SFR of the observed low-metallicity galaxies also explains the observed scattering in X-rays, as shown by Douna et al. (2015). Alternatively, for a given ratio $Q\left(\mathrm{He}^{+}\right) / Q(\mathrm{H})$, the $I(4686) / I(\mathrm{H} \beta)$ intensity may be lower than predicted here (assuming Case B) in nebulae with a low-ionization parameter, as discussed by Raiter et al. (2010) and first explained by Stasińska \& Tylenda (1986).

Some cases of strong He II emission but faint X-ray emission are also known. Such a prominent case is the wellstudied low-metallicity galaxy SBS0335-052E, for example, which shows extended He II $\lambda 4686$ emission with an integrated intensity $I(4686) / I(\mathrm{H} \beta) \approx 0.026$, but faint X-ray emission (see Kehrig et al. 2018; Thuan et al. 2004). The observed value of

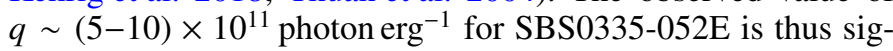
nificantly higher ( $\sim 25-50$ times) than the mean value adopted here, and higher than what is observed in I $\mathrm{Zw} 18$. This casts some doubt on the responsibility of X-rays for the observed He II emission (Kehrig et al. 2018). Interestingly, however, the bulk of the X-ray emission observed with Chandra in SBS0335$052 \mathrm{E}$ is due to a point-like source that is spatially coincident with the brightest super star clusters and the main emission region of He II 14686 . We suggest that beamed X-ray emission could explain the relatively low observed X-ray flux, but produce the observed $\mathrm{He}$ II emission if the $\mathrm{He}^{+}$ionizing photons are absorbed relatively close to the source. If correct, this would imply an intrinsic $L_{X} \sim(0.8-1.6) \times 10^{41} \mathrm{erg} \mathrm{s}^{-1}$, corresponding to several high-mass XRBs or a single ULX. ULXs, and especially those with neutron star accretors, which are expected to slightly dominate older populations (mean stellar population age $>30 \mathrm{Myr}$ ), are expected to be highly beamed source (e.g.,
King 2009; Wiktorowicz et al. 2018), and thus the observed X-ray luminosity per unit of star formation can be a significant underestimate in certain cases.

\subsection{Improvements and future tests}

Although our results show that with the assumption of one parameter $q$, the amount of $\mathrm{He}^{+}$ionizing photons emitted per X-ray luminosity, the high-mass XRB / ULX population can reproduce both the observed nebular He II emission in starforming galaxies and its metallicity dependence, it is difficult to definitely prove the validity of our explanation. Further detailed multiwavelength studies of individual objects, as done for I Zw 18 by Lebouteiller et al. (2017), could provide new insight. Clearly, a next step is to examine in depth the correlation between He II and X-ray emission on an individual object basis, and for the largest possible samples. Future studies should ideally also attempt to predict the spectrum of the XRBs and their emission at low energies to allow more quantitative work that also examines their possible contribution to other emission lines, which are due to stellar photoionization.

Although we suggest that XRBs are the main source of nebular He II $\lambda 4686$ emission at low metallicity, we do not exclude contributions from shocks, as advocated for some galaxies (e.g., Thuan \& Izotov 2005; Izotov et al. 2012). Establishing their role for larger samples and distinguishing between shock and XRB contributions should help to refine our understanding of the highenergy sources in metal-poor star-forming galaxies.

\section{Conclusion}

We have combined results from the detailed multiwavelength study of I Zw 18 from Lebouteiller et al. (2017), which shows that nebular He II $\lambda 4686$ emission in this lowmetallicity galaxy can be explained by an observed massive XRB, with the observed anticorrelation of $L_{X} /$ SFR with metallicity (Douna et al. 2015; Brorby et al. 2016). With this we showed that nebular He II, which is frequently observed in lowmetallicity star-forming galaxies, is most likely due to high-mass XRBs and/or ULX.

Assuming that the bulk of the hydrogen ionizing photons is emitted by normal stars (single or binaries) and that the photons above $54 \mathrm{eV}$ (capable of ionizing $\mathrm{He}^{+}$) are emitted by $\mathrm{X}$-ray binaries, we have shown that the observed anticorrelation of $L_{X} / \mathrm{SFR}$ with $\mathrm{O} / \mathrm{H}$ reproduces the observed dependence of $I(4686) / I(\mathrm{H} \beta)$ on metallicity well (Fig. 1). A single parameter $q$, which describes the ratio of $\mathrm{He}^{+}$ionizing photons per X-ray luminosity emitted from the XRB population and is determined from I Zw 18, suffices to describe this behavior.

To further validate our estimates, we have used the recently recalibrated (Madau \& Fragos 2017) XRB population synthesis models of Fragos et al. (2013a,b), which predict a strong metallicity dependence of the X-ray emission to examine their implications for nebular He II emission. With the same assumptions, we found that these models also predict both the observed $I(4686) / I(\mathrm{H} \beta)$ intensity and its dependence on $\mathrm{O} / \mathrm{H}$ for constant SFRs (Fig. 1). Finally, we predicted the age-dependent He II emission of simple stellar populations at different metallicities by combining the BPASS binary models (Eldridge et al. 2017; Xiao et al. 2018) with the XRB models. Our predictions are in good agreement with the bulk of the observations (see Fig. 3). In some galaxies with very high $\mathrm{H} \beta$ equivalent widths $(W(\mathrm{H} \beta) \gtrsim$ $200 \AA$ ), the observed He II emission is stronger than predicted, 
which may indicate that luminous X-ray sources should appear on very short timescales ( $\lesssim 5 \mathrm{Myr})$.

Overall, we conclude that both empirical data and theoretical models suggest that high-mass X-ray binaries are the main source of nebular He II emission in low-metallicity star-forming galaxies, although contributions from shocks are not excluded.

Acknowledgements. DS wishes to thank Vianney Lebouteiller for exchanges on his modeling of this galaxy. TF is grateful for support from the SNSF Professorship grant (project number PP00P2176868) Y.I. acknowledges support from the National Academy of Sciences of Ukraine (Project No. 0116U003191) and by its Program of Fundamental Research of the Department of Physics and Astronomy (Project No. 0117U000240).

\section{References}

Basu-Zych, A. R., Lehmer, B. D., Hornschemeier, A. E., et al. 2013, ApJ, 774, 152

Berg, D. A., Erb, D. K., Auger, M. W., Pettini, M., \& Brammer, G. B. 2018, ApJ, 859,164

Brorby, M., Kaaret, P., Prestwich, A., \& Mirabel, I. F. 2016, MNRAS, 457, 4081

Cassata, P., Le Fèvre, O., Charlot, S., et al. 2013, A\&A, 556, A68

Douna, V. M., Pellizza, L. J., Mirabel, I. F., \& Pedrosa, S. E. 2015, A\&A, 579, A44

Eldridge, J. J., Stanway, E. R., Xiao, L., et al. 2017, PASA, 34, e058

Fragos, T., Lehmer, B., Tremmel, M., et al. 2013a, ApJ, 764, 41

Fragos, T., Lehmer, B. D., Naoz, S., Zezas, A., \& Basu-Zych, A. 2013b, ApJ, 776, L31

Garnett, D. R., Kennicutt, Jr., R. C., Chu, Y.-H., \& Skillman, E. D. 1991, ApJ, 373,458

Götberg, Y., de Mink, S. E., Groh, J. H., et al. 2018, A\&A, 615, A78

Guseva, N. G., Izotov, Y. I., \& Thuan, T. X. 2000, ApJ, 531, 776

Heap, S. R., Hubeny, I., Bouret, J. C., \& Lanz, T. 2019, in Radiative signatures from the cosmos, eds. K. Werner, \& T. Rauch, ASP Conf. Ser., in press

Izotov, Y. I., \& Thuan, T. X. 1998, ApJ, 500, 188
Izotov, Y. I., \& Thuan, T. X. 2004, ApJ, 602, 200

Izotov, Y. I., Thuan, T. X., \& Privon, G. 2012, MNRAS, 427, 1229

Izotov, Y. I., Guseva, N. G., Fricke, K. J., \& Henkel, C. 2016, MNRAS, 462, 4427

Izotov, Y. I., Thuan, T. X., \& Guseva, N. G. 2018, MNRAS, 483, 5491

Kaaret, P., \& Feng, H. 2013, ApJ, 770, 20

Kaaret, P., Schmitt, J., \& Gorski, M. 2011, ApJ, 741, 10

Kehrig, C., Vílchez, J. M., Pérez-Montero, E., et al. 2015, ApJ, 801, L28

Kehrig, C., Vílchez, J. M., Guerrero, M. A., et al. 2018, MNRAS, 480, 1081

Kennicutt, Jr., R. C. 1998, ARA\&A, 36, 189

King, A. R. 2009, MNRAS, 393, L41

Lebouteiller, V., Péquignot, D., Cormier, D., et al. 2017, A\&A, 602, A45

Lehmer, B. D., Basu-Zych, A. R., Mineo, S., et al. 2016, ApJ, 825, 7

Madau, P., \& Fragos, T. 2017, ApJ, 840, 39

Maeder, A. 1987 , A\&A, 178, 159

Pakull, M. W., \& Angebault, L. P. 1986, Nature, 322, 511EP

Raiter, A., Schaerer, D., \& Fosbury, R. A. E. 2010, A\&A, 523, A64

Rappaport, S. A., Podsiadlowski, P., \& Pfahl, E. 2005, MNRAS, 356, 401

Schaerer, D. 1996, ApJ, 467, L17

Schaerer, D. 2002, A\&A, 382, 28

Schaerer, D. 2003, A\&A, 397, 527

Shirazi, M., \& Brinchmann, J. 2012, MNRAS, 421, 1043

Stanway, E. R., \& Eldridge, J. J. 2019, A\&A, 621, A105

Stark, D. P. 2016, ARA\&A, 54, 761

Stasińska, G., \& Tylenda, R. 1986, A\&A, 155, 137

Stasińska, G., Izotov, Y., Morisset, C., \& Guseva, N. 2015, A\&A, 576, A83

Steidel, C. C., Strom, A. L., Pettini, M., et al. 2016, ApJ, 826, 159

Szécsi, D., Langer, N., Yoon, S.-C., et al. 2015, A\&A, 581, A15

Thuan, T. X., \& Izotov, Y. I. 2005, ApJS, 161, 240

Thuan, T. X., Bauer, F. E., Papaderos, P., \& Izotov, Y. I. 2004, ApJ, 606, 213

Tremmel, M., Fragos, T., Lehmer, B. D., et al. 2013, ApJ, 766, 19

Tumlinson, J., \& Shull, J. M. 2000, ApJ, 528, L65

Tzanavaris, P., Fragos, T., Tremmel, M., et al. 2013, ApJ, 774, 136

van Bever, J., \& Vanbeveren, D. 1998, A\&A, 334, 21

Vanzella, E., De Barros, S., Cupani, G., et al. 2016, ApJ, 821, L27

Wiktorowicz, G., Lasota, J. P., Middleton, M., \& Belczynski, K. 2018, ApJ, submitted [arXiv:1811.08998]

Xiao, L., Stanway, E. R., \& Eldridge, J. J. 2018, MNRAS, 477, 904 